\title{
Knockdown of PSMB7 Induces Autophagy in Cardiomyocyte Cultures: Possible Role in Endoplasmic Reticulum Stress
}

\author{
Victoria O. Kyrychenko ${ }^{a, b}$ Vasyl S. Nagibin ${ }^{a} \quad$ Lesya V. Tumanovska $^{a}$ \\ Denis O. Pashevin ${ }^{a}$ Veronika L. Gurianova ${ }^{a}$ Alexey A. Moibenko ${ }^{a}$ \\ Victor E. Dosenko ${ }^{a}$ Daniel J. Klionsky ${ }^{a, c}$

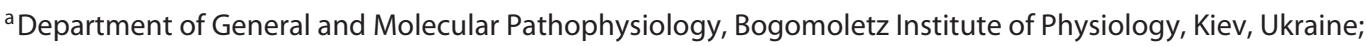 \\ ${ }^{\mathrm{b}}$ Department of Pharmacology and Physiology, UMDNJ-New Jersey Medical School, Newark, NJ, and ' Life Sciences \\ Institute, University of Michigan, Ann Arbor, MI, USA
}

\section{Key Words}

Autophagy - Cardiomyocytes - Endoplasmic reticulum stress · Proteasome $\cdot$ PSMB7 · RNA interference

\begin{abstract}
Proteasomal and autophagic pathways of protein degradation are two essential, endoplasmic reticulum (ER)-associated proteolytic systems involved in the ER stress response. The functional interaction between them has been shown by proteasome pharmacological inhibition. However, little data have been found concerning autophagy induction using an RNA interference approach due to the multisubunit composition of proteolytic systems. We suggested that silencing of single proteasome subunits can induce massive autophagy. Psmb7-specific small interference RNA added to isolated cardiomyocytes significantly affected mRNA expression of essential ER stress marker proteins, including DDIT3/CHOP and HSPA5/GRP78. mRNA expression of the key autophagy regulator MTOR was also increased. These findings were confirmed by single-cell reverse transcription real-time PCR on individual monodansylcadaverine (MDC)labeled cardiomyocytes. RNA interference that decreased the levels of non-catalytic PSMB7 subunits of the protea-
\end{abstract}

some had no influence on chymotrypsin- and trypsin-like activities, but significantly decreased peptidyl-glutamyl peptide-hydrolyzing activity. Immunohistochemical analysis showed increased levels of LC3-marked vacuoles in the cytoplasm of Psmb7-knockdown cells, and MDC staining showed significantly increased numbers of neonatal cardiomyocytes with autophagic vacuoles. After anoxia-reoxygenation, the number of cells with signs of autophagy after Psmb7 gene silencing was higher. Our results indicate that Psmb7 knockdown induces ER stress and autophagy in cardiomyocytes, which may be a useful approach to activate specific autophagy.

Copyright $\odot 2013$ S. Karger AG, Basel

\section{Introduction}

Autophagy and, in some cases, autophagic cell death in eukaryotic cells can be induced by various stimuli, e.g. starvation, amino acid deprivation, rapamycin application and proteasome inhibition [1-3]. The efficiency of autophagy induction differs significantly from case to case and depends on the cell type, dose, duration and many other conditions. Moreover, the same data indicate

\section{KARGER}

E-Mail karger@karger.com

www.karger.com/pat
(C) 2013 S. Karger AG, Base

1015-2008/14/0811-0008\$39.50/0
Dr. Victor E. Dosenko, MD, PhD

Bogomoletz Institute of Physiology

Bogomoletz Street 4

UA-01024 Kiev (Ukraine)

E-Mail dosenko@biph.kiev.ua 
a protective role of autophagy in ischemic injury and against other damaging factors [4-6]. Thus, searching for new methods of autophagy induction is of great theoretical and practical importance.

Proteasomal and autophagic pathways of protein proteolysis are two essential, endoplasmic reticulum (ER)associated degradation systems involved in the ER stress response $[7,8]$. Proteasome inhibition leads to the accumulation of unfolded and polyubiquitinated proteins, autophagosome formation and the induction of autophagy $[9,10]$. Moreover, during the ER stress response, the expression of some specific chaperones, which participate in adaptive rebuilding of misfolded proteins, is increased, especially DDIT3/CHOP, CEBP (CCAAT/enhancer binding protein) and HSPA5/GRP78 (glucose-regulated protein 78) [11]. To date, there are no data about mRNA expression of the above-mentioned proteins in autophagy induction. Theoretically, however, compensatory changes in autophagy-related protein expression upon proteasome inhibition are expected.

RNA interference is an extremely useful method for regulating gene expression, but we have found very few publications concerning autophagy induction that use this approach $[1,12]$. Various studies suggest that degradative systems partially compensate for each other. For example, macroautophagy is upregulated when chaperone-mediated autophagy is inhibited [13]. The functional interaction between proteasomal proteolysis and autophagy accordingly led us to suggest that RNAi-mediated silencing of proteasomal subunits would induce massive autophagy. However, the multicomponent nature and partially replaceable structure of the proteasome render choosing a target gene for knockdown problematic. We decided to focus on proteasome subunit $\beta 7$ (PSMB7) because this subunit is not catalytic and is not replaceable by inducible subunits of the immunoproteasome. Furthermore, PSMB7 is critical for proteasome assembly $[14,15]$.

In our experiments, we knocked down Psmb7 mRNA expression using small interference RNA (siRNA) in primary cultures of neonatal cardiomyocytes, and observed induction of autophagy [monitored by monodansylcadaverine (MDC) staining, immunofluorescence of microtubule-associated protein 1 light chain 3 (LC3) antibody and electron microscopy]. The vast majority of autophagic vacuoles in these cardiomyocytes contained polyubiquitinated proteins. Psmb7 knockdown was followed by a significant increase in Hspa5 mRNA expression in cardiomyocyte cultures, which indicates the onset of ER stress.

Autophagy Induced by PSMB7

Knockdown

\section{Materials and Methods}

\section{Culture of Rat Neonatal Cardiomyocytes}

Neonatal cardiomyocytes were isolated from the ventricular myocardium of 2-day-old Wistar rats by enzymatic digestion according to Reinecke et al. [16] with modifications. The number of living and necrotic cells was determined by staining with $0.2 \%$ trypan blue solution (T6146; Sigma-Aldrich), and was 90-95 and 5-10\%, respectively. Cardiomyocytes were plated in culture dishes covered with $2 \%$ gelatin solution at a density of $120,000 \mathrm{~cm}^{2}$. Cells were cultivated for 1 day in the DMEM/ medium 199 (4:1), supplemented with 7\% calf serum, $4.2 \mathrm{mM}$ $\mathrm{Na}_{2} \mathrm{CO}_{3}, 15 \mathrm{mM}$ HEPES and antibiotics (streptomycin $100 \mathrm{~g} / \mathrm{ml}$, gentamicin $0.05 \mathrm{mg} / \mathrm{ml}$ and penicillin $100 \mathrm{U} / \mathrm{ml}$ ) at $37^{\circ} \mathrm{C}$ in a $5 \%$ $\mathrm{CO}_{2}$ incubator.

\section{RNA Interference}

A double-stranded scrambled (sense $5^{\prime}$-UGU UCA GCG AAA UAU AAC CUU- $3^{\prime}$ and antisense $5^{\prime}$-UUA CAA GUC GCU UUA UAU UGG-3') or Psmb7-specific siRNA (sense $5^{\prime}$-GCU AUU GCA GCU GGC AUC- $3^{\prime}$ and antisense $5^{\prime}$-AGA UGC CAG CUG CAA UAG CUU- $3^{\prime}$ ) was prepared from corresponding oligonucleotides provided by Metabion (Germany) according to the manufacturer's instructions. Freshly isolated cardiomyocytes were washed with sterile PBS and transfected with $2 \mu \mathrm{g}$ of the corresponding siRNAs using the rat cardiomyocytes neo Nucleofector kit (VPE-1002; Lonza, Switzerland) according to the manufacturer's protocol. Immediately after transfection, cells were placed in fresh medium and cultivated for $24 \mathrm{~h}$ as described above.

\section{RNA Isolation, Reverse Transcription and Real-Time PCR}

The cells were harvested for real-time PCR $24 \mathrm{~h}$ after siRNA transfection. Total RNA was isolated from cells using the TRIzol RNA-prep kit (R 1024; Isogen) according to the manufacturer's protocol. RNA concentration was determined using a NanoDrop spectrophotometer ND1000 (NanoDrop Technologies Inc., USA). Reverse transcription was performed using a RevertAid ${ }^{\mathrm{TM}} \mathrm{H}$ minus first-strand cDNA synthesis kit (K1632; Fermentas), using 1.2-1.5 $\mu \mathrm{g}$ of total RNA and random hexamer primer. The cDNA obtained was used for quantitative PCR using primers specific for rat $P s m b 7$, Ddit3/Chop and Hspa5/Grp78 sequences: PSMB7-F 5'-CTG TCC TCA CGG AGA AAG TCA C-3' PSMB7-R 5'-GTC ACC CAG AGA GCT ATC CAA C-3'; CHOP-F $5^{\prime}$-CTT CAC TAC TCT TGA CCC TGC AT-3', CHOP-R 5'-CCG TTT CCT AGT TCT TCC TTG AT-3', GRP78-F 5'-ACC ATG GAG AAA GCT GTA GAG G $-3^{\prime}$, and GRP78-R $5^{\prime}$ - GCC TCC ACT TCC ATA GAG TTT G-3'. mRNA expression was standardized to the Actb (encoding $\beta$-actin) gene, as a housekeeping gene, the transcription levels of which were not influenced under our experimental conditions. The primers used for this were: $\beta$-actin-F $5^{\prime}$-AAG TCC CTC ACC CTC CCA AAA G- $3^{\prime}$ and $\beta$-actin-R $5^{\prime}$-AAG TCC CTC ACC CTC CCA AAA G-3'.

cDNA was separated in $2.5 \%$ agarose gel that contained $10 \mu \mathrm{g} /$ $\mathrm{ml}$ of ethidium bromide. DNA visualization in the gel $(160 \mathrm{~V}, 40$ min) was performed using a transilluminator (Biocom, Russia) and ViTran videosystem. Amplified DNA was subsequently extracted from the gel and purified using a silica bead DNA gel extraction kit (K0513; Fermentas). The concentration of eluted DNA was detected with a NanoDrop spectrophotometer (ND1000; NanoDrop Technologies Inc., USA) and was expressed as the 
number of molecules in $1 \mu \mathrm{l}$. Dilution of purified DNA from $10^{-4}$ to $10^{-8}$ molecules per microliter was used in a calibration curve for quantitative estimation of mRNA expression using real-time PCR.

We performed PCR in $10 \mu \mathrm{l}$ power $\mathrm{SYBR}^{\circledR}$ Green PCR master mix (4368702; Applied Biosystems) containing 40 pM of each primer. The final volume of reaction mix was adjusted to $20 \mu \mathrm{l}$ with deionized water. Thermal cycles started with initial denaturation and AmpliTaq Gold ${ }^{\circledR}$ DNA polymerase activation at $95^{\circ} \mathrm{C}$ for 10 min, followed by treatment at $95^{\circ} \mathrm{C}$ for $15 \mathrm{~s}$ and $58^{\circ} \mathrm{C}$ for $1 \mathrm{~min}$ (45 cycles) followed by a dissociation step. Calculations were performed using the provided 7500 Fast System SDS software. The threshold cycle was defined as the number of cycles required for the fluorescence signal to exceed the detection threshold. We calculated the expression of the target gene relative to the housekeeping gene as the difference between the threshold values of the two genes.

Gene expression of Mtor was determined using the TaqMan gene expression assay (4331182; Applied Biosystems). The pairs of forward and reverse primers for the genes indicated above and the TaqMan probes for the target mRNAs were designed based on the rat mRNA sequence by Applied Biosystems. Gene expression in each probe was normalized to glyceraldehyde-3-phosphate dehydrogenase (Gapdh), as a housekeeping gene, using TaqMan rodent GADPH control reagent (VIC ${ }^{\mathrm{TM}}$ Probe). The thermal cycles of PCR amplification were the following: initial denaturation step at $95^{\circ} \mathrm{C}$ for $20 \mathrm{~s}$, and treatment at $95^{\circ} \mathrm{C}$ for $3 \mathrm{~s}$ and $60^{\circ} \mathrm{C}$ for $30 \mathrm{~s}$ ( 45 cycles) with the use of 7500 Fast Real-Time PCR (Applied Biosystems). Analysis of the data was carried out with 7500 Fast Real-Time PCR Software.

\section{Single-Cell Reverse Transcription Real-Time PCR}

Individual cells from untreated cultures or cultures of rat neonatal cardiomyocytes treated with proteasome inhibitor (clastolactacystin $\beta$-lactone, $5 \mu \mathrm{M}, 1.5 \mathrm{~h}$ ) were first identified by MDC staining for autophagy visualization and then harvested using negative pressure through a glass pipette (4- to 5 - $\mu \mathrm{m}$ tip diameter). The content of each pipette was transferred into a sterile microcentrifuge tube, which contained a lysis solution from the Power SYBR Green Cells-to-CT ${ }^{\text {TM }}$ kit (4402954; Life Technologies), and all of the subsequent procedures were performed according to the manufacturer's manual. The negative controls were run as culture medium without cells. PCR was performed using the Power SYBR Green Cells-to-CT kit, the cycle number was 55, but the annealing temperature was increased by $0.2^{\circ} \mathrm{C}$ during each cycle (from 49 up to $\left.60^{\circ} \mathrm{C}\right)$.

\section{Measurement of Proteasome Activity}

Cells were collected from a Petri dish, sonicated and centrifuged at 5,000 $\mathrm{g}$ for $10 \mathrm{~min}$. The supernatant fraction was incubated in a buffer consisting of $25 \mathrm{~mm}$ Tris- $\mathrm{HCl}$ (pH 7.5) and $1 \mathrm{mM}$ dithiothreitol. The fluorogenic peptide substrate Suc-LeuLeu-Val-Tyr-7-amydo-4-methylcoumarin (S6510; Sigma-Aldrich) was used to measure the chymotrypsin-like activity of the proteasome, Boc-Leu-Ser-Thr-Arg-7-amydo-4-methylcoumarin (B4636; Sigma-Aldrich) was used for the trypsin-like activity and CBZ-Leu-Leu-Glu-7-amydo-4-methylcoumarin (C0483; SigmaAldrich) for the peptidyl-glutamyl peptide-hydrolyzing (PGPH) activity. After a 30-min (for trypsin-like activity) or 1-hour (for other activities) incubation with one of these fluorogenic peptides (at a concentration of $6 \mu \mathrm{M}$ ), fluorescence of the reaction products was monitored at $380 \mathrm{~nm}$ excitation and $440 \mathrm{~nm}$ emission using free 7-amino-4-methylcoumarin as a standard on a Hitachi 4000 spectrofluorometer. The reactions were carried out in the absence or presence of selective proteasome inhibitors - clasto-lactacystin $\beta$-lactone $(2.5 \mu \mathrm{M})$ - to differentiate between nonproteasome- and proteasome-mediated peptide hydrolysis. The percentage of inhibition of respective substrate hydrolysis after treatment with a specific inhibitor was evaluated as the proteasome activity and was expressed as nanomoles per liter 7-amino-4-methylcoumarin per $10^{6}$ cells per $1 \mathrm{~min}$.

\section{Anoxia-Reoxygenation}

After $24 \mathrm{~h}$ of cultivation transfected with scrambled or Psmb7specific siRNA, cells underwent $30 \mathrm{~min}$ of anoxia followed by 60 min of reoxygenation, as described previously [17]. Anoxia was attained with an airtight jar from which $\mathrm{O}_{2}$ was flushed with a gas mixture containing $5 \% \mathrm{CO}_{2}$ and $95 \%$ air. Reoxygenation was realized by exchanging fresh medium and by its aeration with the standard gas mixture.

\section{Fluorescence Microscopy}

Autophagic vacuoles in cardiomyocytes were labeled with 50 $\mu \mathrm{M}$ MDC (D4008; Sigma-Aldrich) at $37^{\circ} \mathrm{C}$ for $10 \mathrm{~min}$. After incubation, cells were washed two times with PBS and immediately analyzed by fluorescence microscopy (Nikon Eclipse E200, filter UV-2A). Four hundred cells were counted from each coverslip. Specificity of MDC staining was examined in the presence of the specific autophagy inhibitor 3-MA (M9281, 5 mM; Sigma-Aldrich) for $24 \mathrm{~h}$ after the electroporation procedure.

For immunofluorescence staining, cells were fixed in $4 \%$ paraformaldehyde in PBS. After fixation, cells were permeabilized with $0.2 \%$ Triton X-100 in PBS for 5 min, and then blocked with $1 \%$ bovine serum albumin in PBS at room temperature for $60 \mathrm{~min}$ followed by overnight incubation with primary rabbit antibodies [LC3 or ubiquitin (L8918 and U5379, respectively);Sigma Aldrich] at $4{ }^{\circ} \mathrm{C}$. After washing with PBS buffer, cells were incubated with FITC-conjugated secondary goat anti-rabbit antibody (F0382; Sigma Aldrich) at room temperature for $2 \mathrm{~h}$. Images were captured using a Nikon Eclipse E200 microscope (filter B-2EC).

\section{Electron Microscopy}

For electron microscopy, cardiomyocytes were plated on NUNC coverslips (150067; Thermanox) and fixed in 2\% paraformaldehyde with $2.5 \%$ glutaraldehyde in $0.1 \mathrm{M}$ phosphate buffer ( $\mathrm{pH} \mathrm{7.4)} \mathrm{for} 2 \mathrm{~h}$ and postfixed in $1 \%$ buffered osmium tetroxide. Then cells were embedded in epoxy resin (45359; Fluka) after dehydration through graded ethanol. Ultrathin sections, doublestained with uranyl acetate and lead citrate, were examined with an electron microscope (Jem-100 CX; Jeol, Japan).

\section{Statistics}

Statistical analysis was performed with Origin 7.0 software. All data were expressed as means $( \pm \mathrm{SE})$ of replicate experiments performed in each assay. Statistical differences were evaluated using ANOVA. $\mathrm{p}<0.05$ was regarded as a statistically significant difference.
Kyrychenko et al. 


\section{Results}

\section{Changes in mRNA Expression upon Psmb7 Silencing}

Administration of Psmb7-specific siRNA to cultured isolated cardiomyocytes induced a 25.5 -fold downregulation of the Psmb7 mRNA level $(\mathrm{p}<0.05)$ compared with scrambled siRNA-treated cells (online supplementary fig. 1; for all online supplementary material, see www. karger.com/doi/10.1159/000350704) that indicates efficient $P s m b 7$ gene silencing. Psmb7 knockdown had a significant effect on mRNA expression of essential ER stress marker proteins, including DDIT3/CHOP and HSPA5/ GRP78. The level of Ddit3 mRNA was increased up to 1.96-fold (online suppl. fig. 2A), and Hspa5 mRNA increased 2.0 -fold ( $\mathrm{p}<0.05$; online suppl. fig. $2 \mathrm{~B}$ ) compared to scrambled siRNA-transfected cells. The level of mRNA expression of the key autophagy regulator MTOR was increased up to 4.34 -fold ( $\mathrm{p}<0.05$; online suppl. fig. $2 \mathrm{C}$ ). This was also confirmed by single-cell reverse transcription real-time PCR on individual MDC-marked cardiomyocytes: the expression of Mtor mRNA was increased $29.8 \%(\mathrm{p}>0.05)$ in spontaneously inducing autophagic cultures and 2.3-fold upon the application of a proteasome inhibitor (clasto-lactacystin $\beta$-lactone). These data indicate that ER stress and a key autophagy-regulatory gene (Mtor) are activated upon Psmb7 knockdown.

\section{Changes in Proteasome Activity upon Psmb7 \\ Knockdown}

To determine whether functional changes in the proteasome active subunits were affected by Psmb7 knockdown, we measured proteasome activities in cell lysates. Trypsin-like activity showed an insignificant decrease after PSMB7 subunit knockdown (27.9 $\pm 5.18 \mathrm{nM} 7$-amino4-methylcoumarin $/ 10^{6}$ cells/min compared to the control with $29.9 \pm 7.16 ; \mathrm{p}=0.53$; online suppl. fig. $3 \mathrm{~A}$ ). In contrast, chymotrypsin-like activity of the proteasome in Psmb7 siRNA-treated cells was $13.1 \pm 1.23$ and remained at the level of scrambled siRNA-treated cells (13.2 \pm 1.73 ; online suppl. fig. 3B). At the same time, we observed a significant reduction in PGPH activity $(0.48 \pm 0.20)$ compared to its activity in scrambled siRNA-transfected cell culture $(1.7 \pm 0.5 ; \mathrm{p}<0.05$; online suppl. fig. $3 \mathrm{C})$.

\section{PSMB7 Subunit Knockdown Activates Autophagy}

siRNA-induced Psmb7 knockdown led to the development of distinctive morphological features of autophagy, which were determined by using MDC staining, 3-methyladenine application, LC3-II immunostaining and electron microscopy. MDC staining showed signifi-

Autophagy Induced by PSMB7

Knockdown cantly increased numbers of neonatal cardiomyocytes with autophagic vacuoles: $36.91 \pm 4.38 \%$ in Psmb7 siRNA-transfected cells compared to control (scrambledtransfected) cells with $23.55 \pm 1.33 \%$ ( $\mathrm{p}<0.05$; online suppl. fig. 4A). Our previous results show an increase in autophagic cell death in a model of anoxia-reoxygenation (AR) in cultures of neonatal cardiomyocytes [4, 18]. There was an even larger increase in the number of autophagic cells after AR in cell culture after Psmb7 gene silencing ( $51.39 \pm 6.2 \%$ ) compared to scrambled siRNAtransfected cells with the AR model $(29.44 \pm 3.02 \%$, p < 0.05 , online suppl. fig. $4 \mathrm{~B})$. AR was used here as a means of additional damage to the cardiomyocytes to investigate whether these cells can manage such injury, which is most common for the heart.

The effect of Psmb7 knockdown with regard to MDC staining (online suppl. fig. 5A) was abolished by 3 -methyladenine $(10 \mathrm{mM})$ added to the culture medium for $24 \mathrm{~h}$ immediately after the placement of transfected cells in culture dishes (online suppl. fig. 5B). The amount of autophagic cells after 3-methyladenine application was not significantly different from scrambled-transfected cells.

To further confirm the effect of $P s m b 7$ downregulation on cell autophagy augmentation, we monitored another parameter of autophagy - LC3-II distribution. LC3 is commonly used as a specific marker of autophagic vacuoles [16]. Immunohistochemical analysis showed extensive localization of LC3-marked vacuoles in the cytoplasm of Psmb7 knockdown cells (online suppl. fig. 6B) in contrast to the uniform distribution of LC3 antibody in control cells (online suppl. fig. 6A).

To determine whether this effect was due to the lack of proteasomal proteolysis, we performed double staining of transfected cells with MDC and ubiquitin antibody. Ubiquitin as a marker for proteasome degradation can accumulate after proteasome inhibition $[19,20]$. We observed colocalization of some ubiquitin aggregates (online suppl. fig. 7B) and MDC-stained vacuoles (online suppl. fig. 7C), which may serve as evidence of their ER accumulation because of the inability to degrade polyubiquitinated proteins by proteasome. In contrast, in scrambled siRNA-treated cells, we observed even antibody distribution without aggregate formation.

The data obtained from electron microscopy also indicate activation of autophagy in cardiomyocytes upon Psmb7 gene silencing. Ultrastructural changes in cells clearly point to the development of ER stress (online suppl. fig. 8). In particular, cells with Psmb7 knockdown revealed ultrastructures that were typical for canonical thapsigargin-induced ER stress [21]. Two different stages 
Table 1. Different changes in cardiomyocyte ultrastructure according to electron-microscopic analysis (online suppl. fig. 8) after Psmb7 RNA interference

\begin{tabular}{llll}
\hline $\begin{array}{l}\text { Online } \\
\text { suppl. fig. }\end{array}$ & Myofilaments & Mitochondria & ER \\
\hline 8A-D & $\begin{array}{l}\text { Partly destroyed, } \\
\text { chaotically presented in } \\
\text { the cytoplasm and } \\
\text { separately located at the } \\
\text { periphery only }\end{array}$ & $\begin{array}{l}\text { Large amount of condensed organelles with } \\
\text { vacuolization. In some cells, they accumulate at the } \\
\text { perinuclear zone } \\
\text { Giant mitochondria appearing and at the same time a } \\
\text { large amount of small, chaotically placed organelles }\end{array}$ & $\begin{array}{l}\text { Insignificant cisterns } \\
\text { swelling in particular at } \\
\text { the perinuclear zone, partial } \\
\text { loss of ribosomes on the } \\
\text { rough ER }\end{array}$ \\
\hline FE, F & Full destruction & $\begin{array}{l}\text { Separated, stretched mitochondria, surrounded by } \\
\text { enlarged ER cisterns }\end{array}$ & $\begin{array}{l}\text { ER cistern swelling, } \\
\text { formation of a large } \\
\text { number of vacuoles }\end{array}$ \\
\hline
\end{tabular}

of cardiomyocyte damage are described in table 1, which reflect the most common organelle modifications against ER remodeling. Along with these changes, there were other signs of cardiomyocyte damage which may be related to ER stress. These included autophagic vacuole accumulation, swelling of the Golgi apparatus, the appearance of multivesicular bodies and the accumulation of lipofuscin in the majority of cells.

\section{Discussion}

Our data show that Psmb7 RNA interference can be used as an effective method for autophagy induction. Our previous work revealed that chemical inhibitors of the proteasome [clasto-lactacystin- $\beta$-lactone ( $\mathrm{cL}$ ), MG132] can induce autophagy in neonatal cardiomyocyte culture, but the number of cells with signs of autophagy did not exceed 25\% [18]. This is in agreement with the data of other authors, which also show compensatory autophagy activation in heart and cardiomyocyte cultures that occurs in response to pharmacologically induced proteasome inhibition by bortezomib [17], MG-262 [22], MG132 and clasto-lactacystin- $\beta$-lactone $[23,24]$. The same effect of proteasome inhibition was shown in many cell types [25-29], where autophagy can be activated through multiple pathways: in response to proteasome inhibitionmediated ER stress to remove ubiquitinated protein aggregates with recruited ubiquitin-binding autophagic receptors such as p62 and NBR1; mTORC1 inhibition [30], and LC3 accumulation [31].

High efficiency of reduction of proteasomal PGPH proteolytic activity using siRNA specific to the mRNA for proteasomal subunit PSMB7 might reflect the key role of this subunit in proteasome self-compartmentalization - incorporation of this subunit triggers dimerization of half-proteasome precursor complexes [14, 15]. Furthermore, its C-terminal extension is an important regulator of the $\beta 1$ subunit active site, which provides PGPH proteasomal activity [14]. Thus, it is possible to assume that reduction of proteasomal activity causes a decrease in the degradation of intracellular proteins, which later accumulate mainly in the ER and can provoke ER stress (an increased level of Ddit3/Chop mRNA in our experiments confirms this hypothesis). Downregulation of the first ER-associated degradation leads to the activation of what can be considered the second ER-associated degradation (i.e. the autophagic machinery) as a compensatory mechanism when proteasomal proteolysis is restricted. An increase in Mtor mRNA expression provides indirect evidence that autophagic processes are activated, because the inactivation of FRAP protein could be expected. Our data indicate that the estimation of Mtor mRNA expression can be used as an additional informative method for autophagy detection, which is in agreement with our previous studies where we observed an increase in mRNA expression for the gene encoding this protein after application of chemical inhibitors of the proteasome [32].

In our experimental settings, induction of autophagy was the most prominent when knockdown of the proteasome subunit PSMB7 was followed by AR. Oxidative damage of proteins after AR certainly requires their rapid elimination by systems of protein degradation [32]. The proteasome degradation upon $\mathrm{AR}$ is restricted due to the high sensitivity of the proteasome to oxidative stress [33] and the ATP dependence of ubiquitination. As a result, compensatory activation of autophagic processes takes place. If one of the proteasome genes is downregulated, the autophagic machinery load increases dramatically during $A R$, leading to a substantial increase in the per-
12

Pathobiology 2014;81:8-14 DOI: $10.1159 / 000350704$
Kyrychenko et al. 
centage of cells with signs of autophagy $(>50 \%)$. We had previously reported that AR induces autophagy in cardiomyocyte cultures, which has a protective effect during this injury [4]. This is consistent with data from other authors concerning the protective role of autophagy during ischemic damage and postinfarction remodeling of the heart $[34,35]$ by removing protein aggregates, which have been found to be increased in number during ischemic heart injury, maintenance of the ATP level and removing damaged mitochondria that can release cytochrome $\mathrm{C}$ by a process called mitophagy, thus reducing apoptotic cell death in the injured area. These studies im- ply that autophagy induction has significant therapeutic potential for decreasing negative consequences of ischemic heart injury.

Thus, RNA-induced silencing of the PSMB7 subunit of the proteasome leads to autophagy induction, accompanied by ER stress activation. Taking into account similar effects of both - chemical inhibitors and siRNA-induced proteasome inhibition on autophagy induction, knockdown of $P s m b 7$ can be used as a method of inducing autophagy accompanied by ER stress-dependent remodeling.

\section{References}

1 Chu C, Shatkin AJ: Apoptosis and autophagy induction in mammalian cells by small interfering RNA knockdown of mRNA capping enzymes. Mol Cell Biol 2008;28:5829-5836.

-2 Schiaffino S, Mammucari C, Sandri M: The role of autophagy in neonatal tissues: just a response to amino acid starvation? Autophagy 2008;4:727-730.

3 Ecker N, Mor A, Journo D, Abeliovich H: Induction of autophagic flux by amino acid deprivation is distinct from nitrogen starvationinduced macroautophagy. Autophagy 2010;6: 879-890.

4 Dosenko VE, Nagibin VS, Tumanovska LV, Moibenko AA: Protective effect of autophagy in anoxia-reoxygenation of isolated cardiomyocytes? Autophagy 2006;2:305-306.

5 Isaka Y, Kimura T, Takabatake Y: The protective role of autophagy against aging and acute ischemic injury in kidney proximal tubular cells. Autophagy 2011;7:1085-1087.

-6 KanamoriH, Takemura G, Goto K, Maruyama R, Ono K, Nagao K, Tsujimoto A, Ogino A, Takeyama T, Kawaguchi T, Watanabe T, Kawasaki M, Fujiwara T, Fujiwara H, Seishima M, Minatoguchi S: Autophagy limits acute myocardial infarction induced by permanent coronary artery occlusion. Am J Physiol Heart Circ Physiol 2011;300:H2261-H2271.

7 Fujita E, Kouroku Y, Isoai A, Kumagai H, Misutani A, Matsuda C, Hayashi YK, Momoi T: Two endoplasmic reticulum-associated degradation (ERAD) systems for the novel variant of the mutant dysferlin: ubiquitin/ proteasome ERAD(I) and autophagy/lysosome ERAD(II). Hum Mol Genet 2007;16: 618-629.

8 Ding WX, Yin XM: Sorting, recognition and activation of the misfolded protein degradation pathways through macroautophagy and the proteasome. Autophagy 2008;4:141-150.

-9 Zhou F, van Laar T, Huang H, Zhang L: APP and APLP1 are degraded through autophagy in response to proteasome inhibition in neuronal cells. Protein Cell 2011;2:377-383.
10 Lonial S, Boise LH: Farnesyl transferase inhibitors, autophagy, and proteasome inhibition: synergy for all the right reasons. Autophagy 2011;7:448-449.

11 Tan YY, Zhou HY, Wang ZQ, Chen SD: Endoplasmic reticulum stress contributes to the cell death induced by UCH-L1 inhibitor. Mol Cell Biochem 2008;318:109-115.

12 Akar U, Chaves-Reyez A, Barria M, Tari A, Sanguino A, Kondo Y, Kondo S, Arun B, Lopez-Berestein G, Ozpolat B: Silencing of Bcl-2 expression by small interfering RNA induces autophagic cell death in MCF-7 breast cancer cells. Autophagy 2008;4:669-679.

13 Kaushik S, Cuervo AM: Chaperone-mediated autophagy: a unique way to enter the lysosome world. Trends Cell Biol 2012;22:407417.

14 Marques AJ, Glanemann C, Ramos PC, Dohmen RJ: The C-terminal extension of the $\beta 7$ subunit and activator complexes stabilize nascent $20 \mathrm{~S}$ proteasomes and promote their maturation. J Biol Chem 2007;282:3486934876.

15 Li X, Kusmierczyk AR, Wong P, Emili A, Hochstrasser M: $\beta$-Subunit appendages promote $20 \mathrm{~S}$ proteasome assembly by overcoming an Ump1-dependent checkpoint. EMBO J 2007;26:2339-2349.

16 Reinecke H, Zhang M, Bartosek T, Charles EM: Survival, integration, and differentiation of cardiomyocyte grafts. Circulation 1999; 100:193-202.

17 Nowis D, Maczewski M, Mackiewicz U, Kujawa M, Ratajska A, Wieckowski MR, Wilczyński GM, Malinowska M, Bil J, Salwa P, Bugajski M, Wójcik C, Siński M, Abramczyk P, Winiarska M, Dabrowska-Iwanicka A, Duszyński J, Jakóbisiak M, Golab J: Cardiotoxicity of the anticancer therapeutic agent bortezomib. Am J Pathol 2010;176:2658-2668.
18 Dosenko VE, Nagibin VS, Tumanovskaya LV, Zagoriy VY, Moibenko AA, Vaage J: Proteasomal proteolysis in anoxia-reoxygenation, preconditioning and postconditioning of isolated cardiomyocytes. Pathophysiology 2006;13:119-125.

19 Klionsky DJ, Abdalla FC, Abeliovich H, et al: Guidelines for the use and interpretation of assays for monitoring autophagy. Autophagy 2012;8:445-544.

20 Gies E, Wilde I, Winget JM, Brack M, Rotblat B, Novoa CA, Balgi AD, Sorensen PH, Roberge M, Mayor T: Niclosamide prevents the formation of large ubiquitin-containing aggregates caused by proteasome inhibition. PLoS One 2010;5:e14410.

21 Tumanovska LV, Nagibin VS, Dosenko VI, Moíbenko OO: Ultrastructural changes in isolated cardiomyocytes in modeling of endoplasmic reticulum stress (in Ukrainian). Fiziol Zh 2008;54:10-21.

22 Zheng Q, Su H, Tian Z, Wang X: Proteasome malfunction activates macroautophagy in the heart. Am J Cardiovasc Dis 2011;1:214-226.

23 Tannous P, Zhu H, Nemchenko A, Berry JM, Johnstone JL, Shelton JM, Miller FJ Jr, Rothermel BA, Hill JA: Intracellular protein aggregation is a proximal trigger of cardiomyocyte autophagy. Circulation 2008;117:3070-3078.

24 Pedrozo Z, Sánchez G, Torrealba N, Valenzuela R, Fernández C, Hidalgo C, Lavandero S, Donoso P: Calpains and proteasomes mediate degradation of ryanodine receptors in a model of cardiac ischemic reperfusion. Biochim Biophys Acta 2010;1802:356-362.

25 Liu C, Yan X, Wang HQ, Gao YY, Liu J, Hu Z, Liu D, Gao J, Lin B: Autophagy-independent enhancing effects of Beclin 1 on cytotoxicity of ovarian cancer cells mediated by proteasome inhibitors. BMC Cancer 2012;12:622.

-26 Ding WX, Ni HM, Gao W, Yoshimori T, Stolz DB, Ron D, Yin XM: Linking of autophagy to ubiquitin-proteasome system is important for the regulation of endoplasmic reticulum stress and cell viability. Am J Pathol 2007;171: 513-524.
Autophagy Induced by PSMB7

Knockdown
Pathobiology 2014;81:8-14 DOI: $10.1159 / 000350704$ 
27 Taylor JM, Brody KM, Lockhart PJ: Parkin co-regulated gene is involved in aggresome formation and autophagy in response to proteasomal impairment. Exp Cell Res 2012;318: 2059-2070.

28 Agholme L, Hallbeck M, Benedikz E, Marcusson J, Kågedal K: Amyloid- $\beta$ secretion, generation, and lysosomal sequestration in response to proteasome inhibition: involvement of autophagy. J Alzheimers Dis 2012;31: 343-358.

29 Selimovic D, Porzig BB, El-Khattouti A, Badura HE, Ahmad M, Ghanjati F, Santourlidis S, Haikel Y, Hassan M: Bortezomib/proteasome inhibitor triggers both apoptosis and autophagy-dependent pathways in melanoma cells. Cell Signal 2013;25:308-318.
30 Ghosh P, Wu M, Zhang H, Sun H: mTORC1 signaling requires proteasomal function and the involvement of CUL4-DDB1 ubiquitin E3 ligase. Cell Cycle 2008;7:373-381.

$31 \mathrm{Wu}$ WK, Wu YC, Yu L, Li ZJ, Sung JJ, Cho $\mathrm{CH}$ : Induction of autophagy by proteasome inhibitor is associated with proliferative arrest in colon cancer cells. Biochem Biophys Res Commun 2008;374:258-263.

32 Surova OV, Nagibin VS, Tumanovskaya LV, Dosenko VE, Moibenko AA: Effect of low dose of proteasome inhibitor on cell death and gene expression in neonatal rat cardiomyocyte cultures exposed to anoxia-reoxygenation. Exp Clin Cardiol 2009;14:e57-e61.
3 Bulteau AL, Lundberg KC, Humphries KM, Sadek HA, Szweda PA, Friguet B: Oxidative modification and inactivation of the proteasome during coronary occlusion/reperfusion. J Biol Chem 2001;276:30057-30063.

34 Hamacher-Brady A, Brady NR, Gottlieb RA: Enhancing macroautophagy protects against ischemia/reperfusion injury in cardiac myocytes. J Biol Chem 2006;281:29776-29787.

35 Nakai A, Yamaguchi O, Takeda T, Higuchi Y, Hikoso S, Taniike M, Omiya S, Mizote I, Matsumura $\mathrm{Y}$, Asahi M, Nishida K, Hori M, Mizushima N, Otsu K: The role of autophagy in cardiomyocytes in the basal state and in response to hemodynamic stress. Nat Med 2007;13:619-624. 